\title{
Molecular Imaging of Cardiovascular Disease Using Ultrasound
}

\author{
Flordeliza S. Villanueva, MD \\ Center for Ultrasound Molecular Imaging and Therapeutics, Cardiovascular Institute, University of \\ Pittsburgh Medical Center, Pittsburgh, PA
}

\section{Introduction}

Ultrasonic detection of molecular markers of cardiovascular disease using acoustically active particles offers the potential for real time in vivo imaging of function-specific, subcellular components of cardiovascular disease with clinically available imaging systems. Molecular imaging with ultrasound utilizes intravascular delivery of microparticles (contrast agents) that are targeted to bind to disease-specific epitopes expressed by endothelial cells. Binding of the contrast agent to the endothelium after systemic injection occurs by virtue of targeting ligands on the microparticle surface which confer specific binding to the endothelial target. In the presence of ultrasound, these bound particles emit an acoustic signal which can be detected as a persistent contrast effect on a two dimensional ultrasound image.

The possibility of molecular imaging using ultrasound is substantiated by a growing body of literature demonstrating proof of concept in vitro and in clinically relevant animal models of disease, lending support to the notion that the "bench to imaging" translation of this technology will be possible in the near future. This purpose of this review is to summarize experimental work in the field of ultrasound molecular imaging, with an emphasis on data that positions this technology to move into clinical application. Properties of ultrasound contrast agents and the principles that govern their detection with ultrasound imaging systems will first be reviewed. Studies proving that ultrasound molecular imaging is possible in vivo will be presented, and finally, a perspective on the requirements for ultimately achieving clinical translation will be discussed.

\section{Properties of ultrasound contrast agents}

Numerous ultrasound contrast agent formulations have been reported (1-8). All the contrast agents have in common the feature that they are micron- to nano- sized particles which produce an ultrasound signal in response to an ultrasound field. This review focuses on the most studied of these contrast agents, gas-filled microspheres ("microbubbles") measuring $2-4 \mu \mathrm{m}$ in diameter. Most commonly, the microbubbles are composed of perfluorocarbon or nitrogen gas encapsulated by shells made of phospholipid, albumin, or biodegradable polymers $(1,2,6,9)$. Because of their size, the microbubbles remain within the intravascular compartment, thus requiring that the molecules being targeted be endoluminal in location.

(C) 2008 American Society of Nuclear Cardiology. Published by Elsevier Inc. All rights reserved.

Address for correspondence: Flordeliza S. Villanueva, MD, Center for Ultrasound Molecular Imaging and Therapeutics, Cardiovascular Institute, University of Pittsburgh Medical Center, A351 Presbyterian University Hospital, 200 Lothrop Street, Pittsburgh, PA 15213, Fax: 412647 4227, Phone: 412647 5840, villanuevafs@ upmc.edu.

Publisher's Disclaimer: This is a PDF file of an unedited manuscript that has been accepted for publication. As a service to our customers we are providing this early version of the manuscript. The manuscript will undergo copyediting, typesetting, and review of the resulting proof before it is published in its final citable form. Please note that during the production process errors may be discovered which could affect the content, and all legal disclaimers that apply to the journal pertain. 
Other ultrasound contrast agents which are not gas-filled microspheres include liposomes (10) and liquid perfluorocarbon emulsion nanoparticles which exit the microvasculture $(4,11)$ and may allow acoustic targeting of extravascular markers.

Microbubble detection using clinical ultrasound imaging systems is based on the fact that at the frequencies used by current ultrasound machines, the microbubbles "resonate" or expand and contract in cyclical fashion (12). At higher acoustic powers, the microbubble oscillations become asymmetric and the microbubbles can be induced to rupture (12). This "non-linear resonance" renders the microbubbles themselves emitters of ultrasound at frequencies that are different from tissue backscatter, resulting in unique acoustic signals distinguishable from tissue background (13). Current ultrasound scanning systems exploit the acoustic "signature" of microbubbles to maximize detection of the contrast agents. The systems vary in transmitted ultrasound frequency, pulse waveform, acoustic pressure, and detection frequency, details of which are reviewed elsewhere (13), but all display the processed microbubble signal as a videointensity increase on the two dimensional image.

\section{Ultrasound contrast agents as red cell tracers: Current clinical applications}

The use of ultrasound contrast agents for molecular imaging is an extension of contrast echocardiographic principles and approaches that are already in clinical practice. Nontargeted ultrasound contrast agents are currently used in echocardiography as red blood cell tracers because they have an intravascular rheology similar to red cells $(9,14)$. Intravenously injected microbubbles are used to opacify the blood pool to enhance endocardial border definition and facilitate more accurate assessment of left ventricular function in technically difficult echocardiograms (15). Two gas-filled microsphere ultrasound contrast agents are currently approved for this indication by the Food and Drug Administration $(1,9)$.

Non-targeted contrast agents are also being developed for myocardial tissue perfusion imaging, in which microbubble transit through the intramyocardial microcirculation causes a transient increase in myocardial tissue backscatter during ultrasound imaging. The resulting time vs backscatter data has been shown to correlate with myocardial blood flow measured using radiolabeled microspheres in animal models $(6,16)$. Furthermore, the regional distribution of microbubbles co-registers with the regional distribution of myocardial blood flow (6). Myocardial contrast echocardiography can detect coronary artery disease $(17,18)$ and myocardial viability $(19,20)$ after infarction. Several commercial microbubble formulations are in clinical testing for perfusion imaging (21).

\section{Ultrasound contrast agents as molecular probes: Principles and proof of concept}

Molecular imaging with microbubbles utilizes their intravascular location and acoustic responsiveness to specifically interrogate molecular features of the endothelial surface. Unlike freely circulating microbubbles used as red cell tracers, targeted microbubbles are engineered to stick to the endothelial surface using specific ligand-receptor interactions. A targeting ligand is attached to the surface of the microbubble, causing the microbubble to adhere to a specific endothelial epitope (Figure 1A). This adhesion manifests in the two dimensional ultrasound image as a videointensity increase in the region where the molecular target is localized, and which persists even after circulating microbubbles have washed out. Alternatively, for inflammation imaging (see below), activated leukocytes can be rendered acoustically active through attachment and phagocytosis of microbubbles. When activated leukocytes with externally attached or internalized microbubbles physiologically adhere to inflamed endothelium, they can be detected with ultrasound as a persistent contrast effect in the region of inflammation (Figure 1B). 
A number of targeting ligands have been successfully used to prove the concept that microbubbles can bind to specific endothelial epitopes. The first of these were monoclonal antibodies direct towards intercellular adhesion molecule -1 (ICAM1), a leukocyte adhesion molecular upregulated by activated endothelial cells. These antibodies were attached to the surface of lipid coated perfluorbutane gas-filled microbubbles and found to adhere to cultured human coronary artery endothelial cells overexpressing ICAM1 (Figure 2) (22). More recently, other targeting moieties have been evaluated, including peptides sequences (23-26) and naturally occurring ligands for the given receptor, including carbohydrates or proteins (27-29). Adhesion of targeted microbubbles is affected by shear stress levels at the vessel wall and receptor density on the endothelial cell (30-32). Ligand density on the microbubbles, ligand-receptor bond affinity, the conformational presentation of the ligand to the receptor, and microbubble shell deformability, are properties that affect net microbubble adhesion and which pose opportunities for optimization of microbubble design (7,30,33-35).

In addition to surface modification of microbubbles so that they present specific targeting ligands to endothelium, leukocytes themselves can become the ultrasound imaging probe by attachment to and ingestion of microbubbles in the setting of inflammation $(36,37)$. It has been shown that activated leukocytes can uptake both lipid and albumin-based microbubbles through complement- and integrin-mediated mechanisms, respectively (Figure 3) (38), and that the microbubbles remain acoustically active even after entry into the leukocyte $(37,39)$.

\section{Clinical applications of ultrasound molecular imaging in cardiovascular disease}

\section{Inflammatory imaging: Ultrasonic detection of leukocyte adhesion molecules}

Leukocyte adhesion molecules are overexpressed by the endothelium of both large arteries as well as the microcirculation in the setting of various cardiovascular diseases (40). Because of their intravascular location, microbubbles have access to leukocyte adhesion molecules expressed by activated endothelium, a property which forms a basis for the ultrasonic diagnosis of cardiovascular disease associated with inflammation.

Ultrasound detection of microvascular adhesion molecule overexpression can be used to detect the "ischemic memory" of myocardium as a means of determining whether symptoms of chest pain are cardiac or non-cardiac in etiology. In this approach, a microbubble is designed to bind to the adhesion molecule P-selectin, which is overexpressed by endothelium within minutes of ischemia/reperfusion, and which persists even after the ischemic event has resolved $(27,41)$. Villanueva et al used a microbubble targeted to bind to $\mathrm{P}$-selectin via the tetrasaccharide sialyl Lewis ${ }^{\mathrm{X}}$, the naturally occurring ligand (27). The targeted microbubbles or control microbubbles bearing sialyl Lewis ${ }^{\mathrm{C}}$ were intravenously administered to rats undergoing transient coronary occlusion followed by reperfusion. Nonlinear ultrasound imaging demonstrated persistent contrast enhancement limited to the risk area, or the region of prior myocardial ischemia (Figure 4). These findings suggest that transthoracic ultrasound imaging utilizing microbubbles that bind to a microvascular endothelial marker of prior ischemia may be useful not only in the etiologic diagnosis of patients presenting with acute or recent chest pain, but also in delineating the extent of myocardium at risk. A similar approach of targeting P-selectin was reported by Lindner and colleagues in which microbubbles with anti-P selectin antibody on the surface were used to ultrasonically image post ischemic inflammation in murine models of transient renal (12) and coronary artery (40) occlusion.

Another application of inflammatory imaging based on leukocyte adhesion molecule overexpression is the detection of acute heart transplant rejection using microbubbles targeted to bind to the ICAM1. Microbubbles with anti-ICAM1 antibodies on their surface 
were intravenously injected into rats with recent allograft or isograft heterotopic cardiac transplantation, with and without acute rejection, respectively (43). Intravenous injection of ICAM1 targeted microbubbles caused persistent, diffuse myocardial contrast enhancement during ultraharmonic imaging of allografted, rejecting myocardium, which was not seen after injection of nonsense-targeted microbubbles (Figure 5). Such data suggest that targeted contrast ultrasound could be useful in the non-invasive diagnosis of acute organ transplant rejection using routine two-dimensional echocardiography, thus precluding the requirement for invasive endomyocardial biopsy.

In addition to the molecular interrogation of the microvascular endothelium, ultrasonic probing for leukocyte adhesion molecule overexpression can be attempted in the larger arteries, such as the coronary or carotid arteries. Such an approach may enable detection of inflammatory events associated with early atherogenesis. Atherosclerosis is heralded by endothelial dysfunction, intimal xanthoma, and pathologic intimal thickening, a molecular hallmark of which includes the overexpression of adhesion molecules that mediate leukocyte infiltration of the endothelium (40). The strategies described above for microbubble design and imaging of microvascular leukocyte adhesion molecules can be extended to the preclinical detection of atherosclerotic plaques, and/or atherosclerosis-prone endothelium in larger vessels. The identification of leukocyte adhesion molecules on endothelium of coronary or peripheral arteries could be the basis for early disease detection (before luminal stenoses develop), and serve to mark "vulnerable patients" at risk for atherosclerotic cardiovascular disease even prior to clinical events, and hence needing aggressive primary preventative treatment.

Ultrasound molecular imaging of the larger arteries for plaque characterization and/or risk assessment of lesions and patients as suggested above poses additional challenges not encountered in molecular imaging of the microcirculation. The issues here pertain to the sensitivity for detection of adhered microbubbles and the spatial resolution of the systems used for vascular imaging. With microvascular molecular imaging in the applications described above, the adhered microbubbles are present collectively in relatively high concentration per myocardial tissue mass due to adhesion within a matrix of intramyocardial microvessels imaged together in the ultrasound beam. In contrast, targeted microbubble adhesion to distinct molecular components of atherosclerotic plaque in larger vessels is inherently limited to the two-dimensional plane of the early atherosclerotic lesion, with the net result being fewer microbubbles per tissue mass in the ultrasound beam, and a diminished acoustic signal.

Another important requirement for molecular imaging of larger arteries is the appropriate coupling of the ultrasound imaging frequency with the natural resonance frequency of the microbubble, such that microbubble "harmonics" or non-linear behavior, which enable the detection of the microbubble-specific signal, are elicited. This resonant frequency is fortuitously the frequency used in clinical echocardiography (1-3 MHz), such that molecular imaging of intramyocardial microcirculation is possible in this frequency range. However, for vascular imaging, higher frequencies are required in order to achieve adequate near-field spatial resolution, such as for coronary intravascular ultrasound (IVUS, $30-40 \mathrm{MHz}$ ) or surface vascular ultrasound (7-12 MHz). As these frequency ranges are not within the natural resonance frequency of 2 to $4 \mu \mathrm{m}$-sized microbubbles, the conventional signal detection strategy for microbubbles is more challenging when they are adhered to endothelium of larger vessels.

Despite these issues, there have been promising developments on the microbubble and imaging system ends which open possibilities for ultrasound molecular imaging of plaque components in larger vessels. Acoustically active lipid particles ("immunoliposomes") 
conjugated to antibodies against ICAM1, vascular cell adhesion molecule (VCAM), fibrinogen, fibrin, and tissue factor increased ultrasound backscatter from atheromatous endothelium during IVUS imaging in a porcine model of atherosclerosis (10). Due to the considerations cited above, however, the signal to noise ratio of this approach has been a limitation, as has the requirement for direct intra-arterial injection to achieve adequate particle adhesion.

Recently, more robust acoustic detection of early atherosclerosis was reported using contrast agents targeted to adhesion molecules and non-linear high frequency ultrasound imaging of a spectrum of early lesions in apo-E deficient vs. wild type mice fed chow vs.

hypercholesterolemic diets (44). Microscopically confirmed aortic adhesion of intravenously injected lipid microbubbles bearing anti-VCAM antibodies was accompanied by persistent ultrasound contrast enhancement of the aorta (Figure 6). Additionally, the degree of videointensity change was related to the extent of plaque formation, suggesting that VCAMtargeted microbubbles can both detect and quantify inflammation in early atherosclerosis. These data prove the concept that leukocyte adhesion molecule detection by ultrasound is possible in large arteries with early atheroma. Extension of this approach, for example, to carotid imaging in clinical populations could ultimately allow in vivo, in situ detection of biomarkers of cardiovascular risk, although such an approach remains to be tested.

\section{Inflammatory imaging: Ultrasonic detection of activated leukocytes}

The ability of leukocytes to internalize microbubbles and the retention of acoustic activity after microbubble phagocytosis $(36,37,39)$ have been utilized to detect post-ischemic tissue in ischemia-reperfusion models, in which leukocyte activation are prominent features. Lipid microbubbles augmented with phosphatidyl serine to facilitate complement-mediated interactions between the microbubble and leukocytes were intravenously injected into canines undergoing transient coronary occlusion of sufficient duration to cause infarction, followed by reperfusion (45). During reperfusion, persistent contrast enhancement was noted in the risk area, and co-localized with the distribution of activated leukocytes (Figure 7). These data suggest when there is intense leukocyte activation, inflammatory imaging with ultrasound can be performed without the requirement for coupling of a targeting ligand to the microbubble.

\section{Angiogenesis imaging}

Ultrasound molecular imaging has the potential for assessing the efficacy of therapeutic angiogenesis. Clinical trials of therapeutic angiogenesis in patients with ischemic heart disease have demonstrated less robust results than were predicted based on animal studies, when traditional clinical metrics such as exercise tolerance and nuclear SPECT imaging have been used to gauge therapeutic response (46). It has been suggested that a molecular angiogenesis marker could provide a useful, more sensitive measure for identifying a biological effect in patients undergoing therapeutic angiogenesis (46). Microbubbles targeted to $a_{v} \beta_{3}$ integrins via have been peptides or to vascular endothelial growth factor (VEGF) receptors via $\mathrm{VEGF}_{121}$ reported to identify angiogenic microcirculation in animal models of tumor- or growth factor-mediated angiogenesis (24-26,28). A tripeptide sequence (arginine-arginine-leucine, RRL), identified with a bacterial peptide display library panned against squamous cell tumor-derived endothelial cells was attached to a microbubble and shown to adhere specifically to angiogenic tumor endothelium (Figure 8) in nude mice with experimentally created sarcoma and prostate tumors (23). In a rat model of malignant glioma, microbubble targeting to $a_{v} \beta_{3}$ integrins via the peptide echistatin enabled identification and tracking of the time course of tumor angiogenesis (26). Such concepts can be extended to echocardiographic imaging for myocardial angiogenesis in response to 
therapeutic interventions, and may provide a rational basis for tailoring such therapies to patients who demonstrate the most robust response based on molecular imaging data.

\section{Future directions}

The concept that targeted ultrasound contrast agents designed to adhere to endothelial epitopes can be detected with ultrasound scanning systems that are already clinically available has clearly been established. Contrast echocardiography using standard, nontargeted microbubbles has already been used in myocardial applications whereby the intramyocardial microvasculare is the compartment that is interrogated. As such, the strategies for molecular imaging with ultrasound were developed initially in models of microvascular disease, such as tissue inflammation following ischemia and during heart transplant rejection, and with angiogenesis. Nonetheless, extension of this technology to the evaluation of the molecular markers of disease in larger vessels also appears to be feasible. The non-invasive nature of ultrasound technology, the lack of radioisotope requirement, and the portability and widespread availability of ultrasound machines compared to nuclear or magnetic scanners, render ultrasound molecular imaging clinically appealing.

The studies discussed above affirm that molecular imaging with ultrasound is possible in animal models of disease. Collaborative efforts from multiple disciplines to address several critical challenges will be required to successfully navigate the transition from the experimental laboratory to the patient bedside. Improvement in signal to noise ratio is one of the core challenges, and will require enhancements in the contrast agent itself (microbubble chemistry) as well in the mechanisms to manipulate and detect the agent (transducers, imaging systems). Optimization of microbubble design and formulation are needed to increase adhesion to the target in order to maximize the signal. Such an effort requires identification of physiologically and clinically appropriate, "image friendly" endothelial targets (high expression). In addition, there is a need to identify strongly binding targeting ligands which can be chemically coupled to the contrast agent without altering binding properties, and which will confer specific, persistent microbubble adhesion. The design of a microbubble with "ideal" binding characteristics, including configuration of relative proportions of ligands if multi-targeting agents are synthesized, requires a combination of kinetic modeling and iterative empiric testing to yield results in the most efficient manner. Such efforts at contrast agent optimization may also include creation of new "non-bubble" contrast agents $(4,10,11)$ or acoustically active modified microbubble formulations $(7)$. Ultrasound manipulation of microbubble kinetics through use of acoustic radiation force to "push" microbubbles to their target may be another approach to increasing microbubble binding events (47).

In addition, strategies for microbubble detection need to be optimized specifically for molecular imaging applications, in which relatively small numbers of adhered bubbles must be detected above and beyond background tissue noise and signals from non-adhered microbubbles. The optimal imaging system should operate on a detection strategy that reflects an understanding of distinct acoustic behaviors of adhered microbubbles (48-50) acquired through systematic optical and acoustic characterization of microbubble formulations under various ultrasound conditions.

For coronary or peripheral arterial imaging, microbubble signals must be detectable in the higher frequency range required for intravascular or surface vascular imaging. In addition to new microbubble designs, new transducers would need to be built to detect microbubble resonance at higher frequencies. Recently, a "harmonic" IVUS transducer was designed which can detect adventitial contrast-enhanced adventitial vasa vasorum in atherosclerotic rabbit aortas (51). If IVUS approaches to detect non-linear microbubble behavior prove to 
be robust, and if microbubbles can be developed to bind to markers of plaque vulnerability, then there is even the exciting possibility of differentiating rupture risk, and hence customizing treatment, among non-occlusive plaques identified at the time of coronary angiography.

\section{Conclusions}

Our increasing insight of the mechanisms of disease on a sub-cellular level has created a need to visualize sub-cellular events in clinical populations in order for treatment paradigms to advance. The evolution of molecular imaging illustrates the contemporary challenges of moving novel diagnostic technologies from robust laboratory proof of concept to clinical demonstration of feasibility and efficacy in an era of molecular medicine. As with other molecular imaging strategies being developed for patient use, ultrasound molecular imaging inherently commands that a well orchestrated, multidisciplinary effort be entrained to successfully move this technology from the bench to the clinical arena.

\section{Acknowledgments}

Dr. Villanueva is supported in part by a grant (RO1HL077534) from the National Institutes of Health

\section{References}

1. Skyba DM, Camarano G, Goodman NC, Price RJ, Skalak TC, Kaul S. Hemodynamic characteristics, myocardial kinetics and microvascular rheology of FS-069, a second generation echocardiographic contrast agent capable of producing myocardial opacification from a venous injection. J Am Coll Cardiol. 1996; 28:1292-1300. [PubMed: 8890829]

2. Huang S, Hamilton AJ, Nagaraj A, Tiukinhoy SD, Klegerman ME, McPherson DD, et al. Improving ultrasound reflectivity and stability of echogenic liposomal dispersions for use as targeted ultrasound contrast agents. J Pharmacol Sci. 2001; 90(12):1917-26.

3. Demos SM, Alkan-Onyuksel H, Kane BJ, Ramani K, Nagaraj A, Greene R, et al. In vivo targeting of acoustically reflective liposomes for intravascular and transvascular ultrasonic enhancement. J Am Coll Cardiol. 1999; 33:867-75. [PubMed: 10080492]

4. Lanza GM, Abendschein DR, Hall CS, Marsh JN, Scott MJ, Scherrer DE, et al. Molecular imaging of stretch-induced tissue factor expression in carotid arteries with intravascular ultrasound. Invest Radiol. 2000; 35:227-34. [PubMed: 10764091]

5. Straub JA, Chickering DE, Hartman TG, Gloff CA, Bernstein H. AI-700 pharmacokinetics, tissue distribution and exhaled elimination kinetics in rats. International Journal of Pharmaceutics. 2007; 328:35-41. [PubMed: 16950578]

6. Villanueva FS, Gertz EW, Csikari M, Pulido G, Fisher D, Sklenar J. Detection of coronary artery stenosis using power Doppler imaging. Circulation. 2001; 103:2624-30. [PubMed: 11382734]

7. Rychak JJ, Lindner JR, Ley K, Klibanov AL. Deformable gas-filled microbubbles targeted to Pselectin. J Controlled Release. 2006; 114:288-99.

8. Fisher NG, Christiansen JP, Leong-Poi H, Jayaweera AR, Lindner JR, Kaul S. Myocardial and microcirculatory kinetics of BR14, a novel third-generation intravenous ultrasound contrast agent. J Am Coll Cardiol. 2002; 39:530-7. [PubMed: 11823094]

9. Lindner JR, Song J, Jayaweera AR, Sklenar J, Kaul S. Microvascular rheology of Definity microbubbles after intra-arterial and intravenous administration. J Am Soc Echo. 2002; 15:396-403.

10. Hamilton AJ, Huang SL, Warnick D, Rabbat M, Kane B, Nagaraj A, et al. Intravascular ultrasound molecular imaging of atheroma components in vivo. J Am Coll Cardiol. 2004; 43:453-60. [PubMed: 15013130]

11. Marsh JN, Hall CS, Scott MJ, Fuhrhop RW, Gaffney PJ, Wickline SA, et al. Improvements in the ultrasonic contrast of targeted perfluorocarbon nanoparticles using an acoustic transmission line model. IEEE Trans Ultra Ferr Freq Control. 2002; 49:29-38. 
12. de Jong N, Bouakaz A, Frinking P. Basic acoustic properties of microbubbles. Echocardiography. 2002; 12:229-40. [PubMed: 12022933]

13. Becher, H.; Burns, PN. LV function and myocardial perfusion. Springer-Verlag; Berlin Heidelberg New York: 2000. Handbook of Contrast Echocardiography.

14. Jayaweera AR, Edwards N, Glasheen WP, Villanueva FS, Abbott RD, Kaul S. In vivo myocardial kinetics of air-filled albumin microbubbles during myocardial contrast echocardiography: comparison with radiolabeled red blood cells. Circ Res. 1994; 74:1157-65. [PubMed: 8187282]

15. Hundley WG, Kizilbash AM, Afridi I, Franco F, Peshock R, Grayburn PA. Administration of an intravenous perfluorocarbon contrast agent improves echocardiographic determination of left ventricular volumes and ejection fraction: comparison with cine magnetic resonance imaging. J Am Coll Cardiol. 1998; 32:1426-32. [PubMed: 9809958]

16. Wei K, Jayaweera AR, Firoozan S, Linka A, Skyba DM, Kaul S. Quantification of myocardial blood flow with ultrasound-induced destruction of microbubbles administered as a constant venous infusion. Circulation. 1998; 97:473-8. [PubMed: 9490243]

17. Wei K, Tong KL, Belcik T, Rafter P, Ragosta M, Wang XQ, et al. Detection of coronary stenoses at rest with myocardial contrast echocardiography. Circulation. 2005; 112:1154-60. [PubMed: 16103241]

18. Elhendy A, O'Leary EL, Xie F, McGrain AC, Anderson JR, Porter TR. Comparative accuracy of real time myocardial contrast perfusion imaging and wall motion analysis during dobutamine stress echocardiography for the diagnosis of coronary artery disease. J Am Coll Cardiol. 2004; 44:2185-91. [PubMed: 15582317]

19. Ragosta M, Camarano G, Kaul S, Powers ER, Sarembock IJ, Gimple LW. Microvascular integrity indicates myocellular viability in patients with recent myocardial infarction. New insights using myocardial contrast echocardiography. Circulation. 1994; 89:2562-69. [PubMed: 8205665]

20. Dwivedi G, Janardhanan R, Hayat SA, Swinburn JM, Senior R. Prognostic value of myocardial viability detected by myocardial contrast echocardiography early after acute myocardial infarction. J Am Coll Cardiol. 2007; 50:327-34. [PubMed: 17659200]

21. Wei K, Crouse L, Weiss J, Villanueva F, Schiller NB, Naqvi TZ, et al. Comparison of usefulness of dipyridamole stress myocardial contrast echocardiography to technetium-99m sestamibi singlephoton emission computed tomography for detection of coronary artery disease (PB127 Multicenter Phase 2 Trial results). Am J Cardiol. 2003; 91:1293-8. [PubMed: 12767419]

22. Villanueva FS, Jankowski RJ, Klibanov S, Pina ML, Alber SM, Watkins SC, et al. Microbubbles targeted to intercellular adhesion molecule-1 bind to activated coronary artery endothelial cells: a novel approach to assessing endothelial function using myocardial contrast echocardiography. Circulation. 1998; 98:1-5. [PubMed: 9665051]

23. Weller GER, Wong MKK, Modzelewski RA, Lu E, Klibanov AL, Wagner WR, et al. Ultrasonic imaging of tumor angiogenesis using contrast microbubbles targeted via the tumor-binding peptide RRL. Cancer Res. 2005; 65:533-9. [PubMed: 15695396]

24. Leong-Poi H, Christiansen J, Klibanov AL, Kaul S, Lindner JR. Noninvasive assessment of angiogenesis by ultrasound and microbubbles targeted to $\mathrm{a}_{\mathrm{v}}$-integrins. Circulation. 2003; 107:455-60. [PubMed: 12551871]

25. Leong-Poi H, Christiansen J, Heppner P, Lewis CW, Klibanov AL, Kaul S, et al. Assessment of endogenous and therapeutic arteriogenesis by contrast ultrasound molecular imaging of integrin expression. Circulation. 2005; 111:3248-54. [PubMed: 15956135]

26. Ellegala DB, Leong-Poi H, Carpenter JE, Klibanov AL, Kaul S, Shaffrey ME, et al. Imaging tumor angiogenesis with contrast ultrasound and microbubbles targeted to $a_{v} a_{3}$. Circulation. 2003; 108:336-41. [PubMed: 12835208]

27. Villanueva FS, Lu E, Bowry S, Kilic S, Tom E, Wang J, et al. Myocardial ischemic memory imaging using molecular echocardiography. Circulation. 2007; 115:345-52. [PubMed: 17210843]

28. Wang J, Kilic S, Tom E, Lu E, Schellenberger U, Schreiner FG, et al. Vascular endothelial growth factor-conjugated ultrasound microbubbles adhere to angiogenic receptors. Circulation. 2005; 112:II-502. (abstract). 
29. Rychak JJ, Li B, Acton ST, Leppanen A, Cummings RD, Ley K, Klibanov AL. Selectin ligands promote ultrasound contrast agent adhesion under shear flow. Mol Pharmaceutics. 2006; 3:51624.

30. Weller GER, Villanueva FS, Klibanov AL, Wagner WR. Modulating targeted adhesion of an ultrasound contrast agent to dysfunctional endothelium. Ann Biomed Eng. 2002; 30:1012-19. [PubMed: 12449762]

31. Weller GE, Villanueva FS, Tom EM, Wagner WR. Targeted ultrasound contrast agents: in vitro assessment of endothelial dysfunction and multi-targeting to ICAM-1 and sialyl Lewis ${ }^{\mathrm{X}}$. Biotechnol Bioeng. 2005; 92:780-8. [PubMed: 16121392]

32. Takalkar AM, Klibanov AL, Rychak JJ, Lindner JR, Ley K. Binding and detachment dynamics of microbubbles targeted to P-selectin under controlled shear flow. J Controlled Release. 2004; 96:473-82.

33. Klibanov AL. Microbubble contrast agents: Targeted ultrasound imaging and ultrasound- assisted drug-delivery applications. Invest Radiol. 2006; 41:354-62. [PubMed: 16481920]

34. Klibanov AL, Rychak JJ, Yang WC, Alikhani S, Li B, Acton S, et al. Targeted ultrasound contrast agent for molecular imaging of inflammation in high-shear flow. Contrast Media \& Molecular Imaging. 2006; 1:259-66. [PubMed: 17191766]

35. Leong-Poi H, Song J, Rim SJ, Christiansen J, Kaul S, Lindner JR. Influence of microbubble shell properties on ultrasound signal: Implications for low-power perfusion imaging. J Am Soc Echo. 2002; 15:1269-76.

36. Lindner JR, Long J, Xu F, Klibanov AL, Singbartl K, Ley K, et al. Noninvasive ultrasound imaging of inflammation using microbubbles targeted to activated leukocytes. Circulation. 2000; 102:2745-50. [PubMed: 11094042]

37. Linder JR, Dayton PA, Coggins MP, Ley K, Song J, Ferrara K, et al. Noninvasive imaging of inflammation by ultrasound detection of phagocytosed microbubbles. Circulation. 2000; 102:5318. [PubMed: 10920065]

38. Lindner JR, Coggins MP, Kaul S, Klibanov AL, Brandenburger GH, Ley K. Microbubble persistence in the microcirculation during ischemia/reperfusion and inflammation is caused by integrin- and complement-mediated adherence to activated leukocytes. Circulation. 2000; 101:668-75. [PubMed: 10673260]

39. Dayton PA, Chomas JE, Lum AF, Allen JS, Lindner JR, Simon SI, et al. Optical and acoustical dynamics of microbubble contrast agents inside neutrophils. Biophys J. 2001; 80:1547-56. [PubMed: 11222315]

40. Libby P. Inflammation in atherosclerosis. Nature. 2002; 450:868-74. [PubMed: 12490960]

41. Kaufmann BA, Lewis C, Xie A, Mirza-Mohd A, Lindner JR. Detection of recent myocardial ischaemia by molecular imaging of P-selectin with targeted contrast echocardiography. Eur Heart J. 2007; 28:2011-7. [PubMed: 17526905]

42. Lindner JR, Song J, Christiansen J, Klibanov AL, Xu F, Ley K. Ultrasound assessment of inflammation and renal tissue injury with microbubbles targeted to p-selectin. Circulation. 2001; 104:2107-12. [PubMed: 11673354]

43. Weller GE, Lu E, Csikari MM, Klibanov AL, Fischer D, Wagner WR, et al. Ultrasound imaging of acute cardiac transplant rejection with microbubbles targeted to intercellular adhesion molecule-1. Circulation. 2003; 108:218-24. [PubMed: 12835214]

44. Kaufmann BA, Sanders JM, Davis C, Xie A, Aldred P, Sarembock IJ, et al. Molecular imaging of inflammation in atherosclerosis with targeted ultrasound detection of vascular cell adhesion molecule-1. Circulation. 2007; 116:276-84. [PubMed: 17592078]

45. Christiansen JP, Leong-Poi H, Klibanov AL, Kaul S, Lindner JR. Noninvasive imaging of myocardial reperfusion injury using leukocyte-targeted contrast echocardiography. Circulation. 2002; 105:1764-7. [PubMed: 11956115]

46. Simons M. Angiogenesis: Where do we stand now? Circulation. 2005; 111:1556-1566. [PubMed: 15795364]

47. Rychak JJ, Klibanov AL, Ley KF, Hossack JA. Enhanced targeting of ultrasound contrast agents using acoustic radiation force. Ultrasound Med Biol. 2007; 33:1132-9. [PubMed: 17445966] 
48. Lanford M, Behm C, Yeh J, Klibanov A, Robinson P, Lindner J. Effect of microbubble ligation to cells on ultrasound signal enhancement: implications for targeting imaging. Invest Radiol. 2006; 41:721-8. [PubMed: 16971795]

49. Zhao S, Kruse DE, Ferrara KW, Dayton PA. Selective imaging of adherent targeted ultrasound contrast agents. Phys Med Biol. 2007; 52:2055-72. [PubMed: 17404455]

50. Borden MA, Sarantos MR, Stieger SM, Simon SI, Ferara KW, Dayton PA. Ultrasound radiation force modulates ligand availability on targeted contrast agents. Mol Imaging. 2006; 5:139-47. [PubMed: 16954028]

51. Goertz DE, Frijlink ME, Tempel D, van Damme LCA, Krams R, Schaar JA, et al. Contrast harmonic intravascular ultrasound. A feasibility study for vasa vasorum imaging. Invest Radiol. 2006; 41:631-8. [PubMed: 16829746] 
A.

\section{Endothelial Cell Imaging}

\section{Ligand}

4 Y Disease-specific

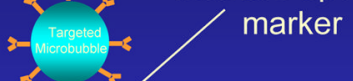

d

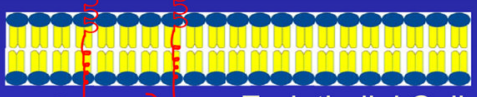

Endothelial Cell

Membrane
B.

\section{Leukocyte Imaging}

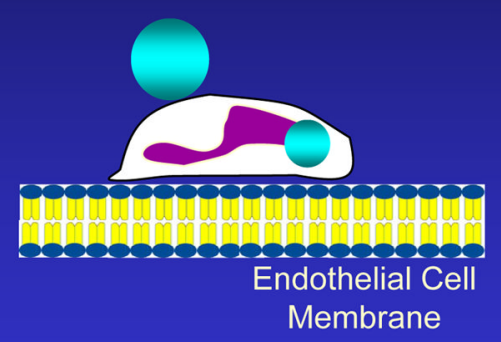

Figure 1.

Schematic diagram of vascular endothelial lining and approaches for ultrasound molecular imaging in which ultrasound contrast agents, or microbubbles, adhere to endothelium. A. Microbubbles with a targeting ligand on their surface can bind specifically to a molecule overexpressed by endothelium in cardiovascular disease, such as a leukocyte adhesion molecule. B. Activated leukocytes may bind or phagocytose microbubbles, which remain acoustically active for a brief period of time, allowing ultrasound detection of leukocytes which have adhered to inflammatory endothelium. Figure not drawn to scale. 

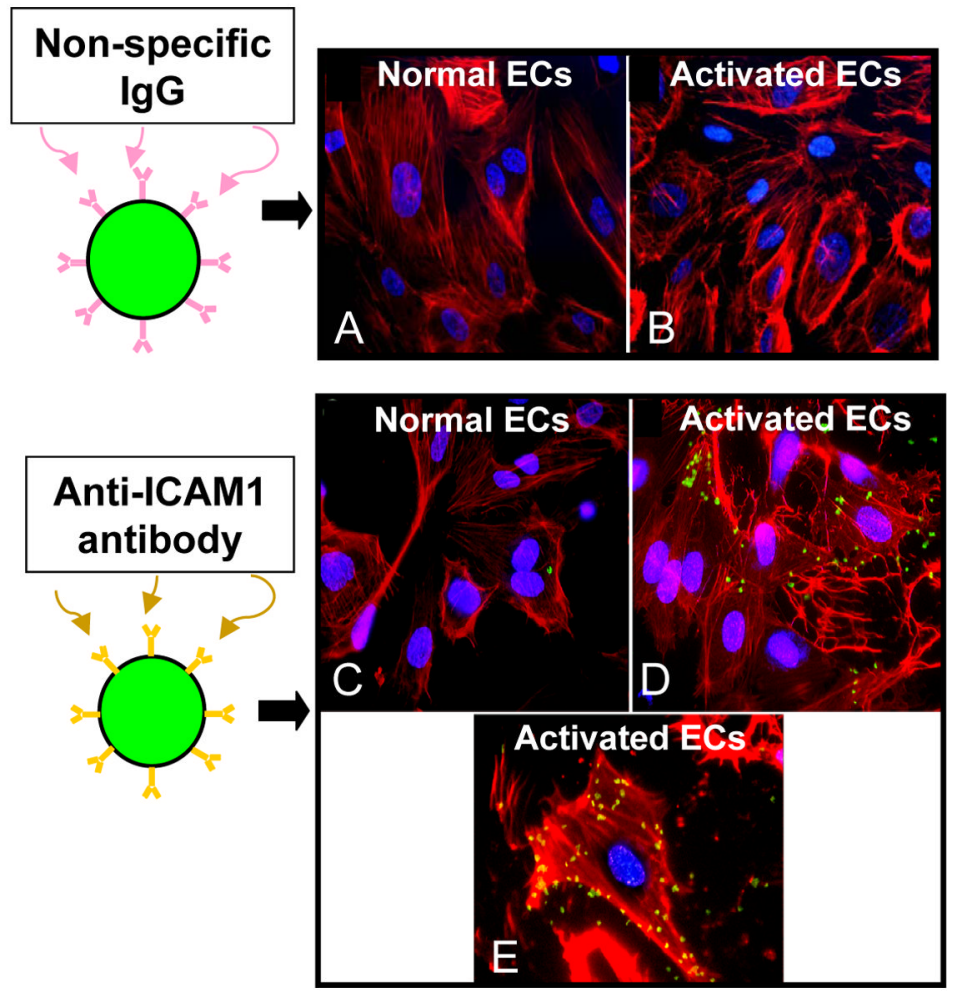

Figure 2.

In vitro studies demonstrating proof of the concept that microbubbles can adhere to a biological surface via a specific targeting ligand. Fluorescent microbubbles (green) were conjugated to either non-specific IgG (control) or to antibody directed against intercellular adhesion molecule 1 (ICAM1) and allowed dwell with cultured human coronary artery endothelial cells (ECs, F-actin labeled with rhodamine, red). Control microbubbles did not adhere to cells at baseline (A) or after endothelial activation to overexpress ICAM1 (B). Anti-ICAM1 microbubbles adhered minimally to non-activated cells due to constitutive expression of ICAM1 (C), and adhered abundantly to activated endothelial cells (D). Panel $\mathrm{E}$ is a higher power image of a single activated cell to which multiple ICAM1-targeted microbubbles have adhered. Reprinted with permission from Ref 22 


\section{Albumin}
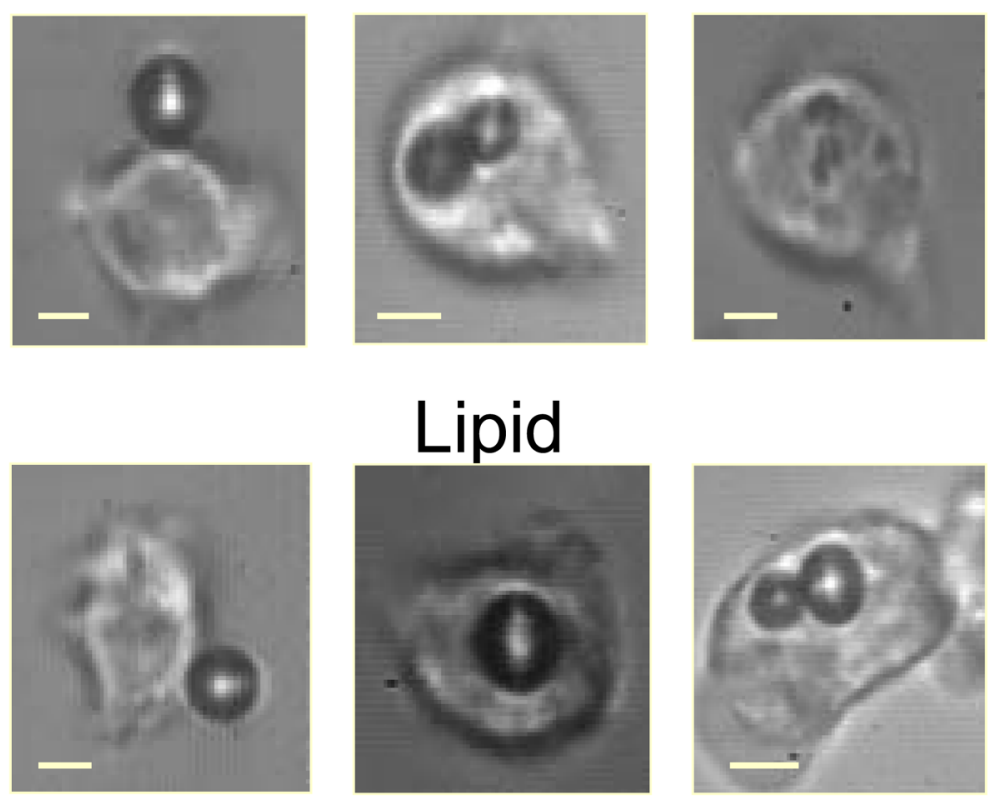

\section{$3 \min$}

$15 \min$

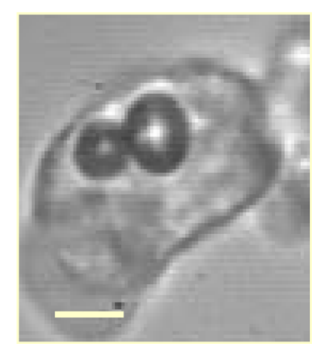

Figure 3.

Photomicrographs demonstrating adhesion of microbubbles made of albumin or lipid shells to activated leukocytes 3 minutes after exposure, and the time course of subsequent phagocytosis. Reprinted with permission from Ref 37. 


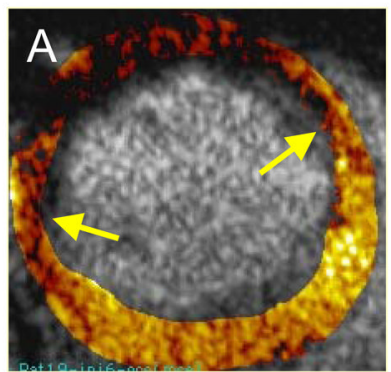

Occlusion

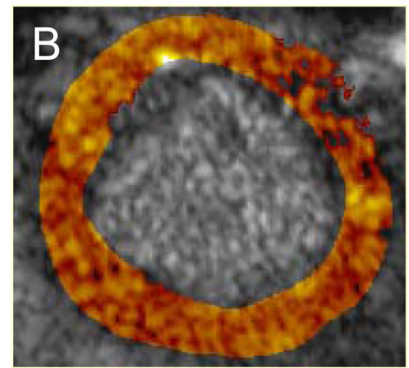

Reperfusion

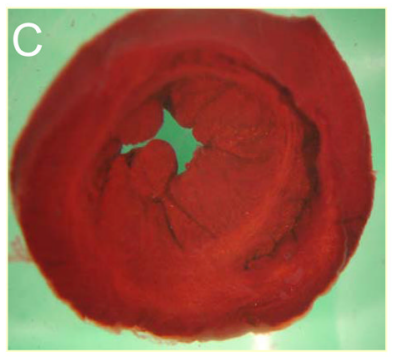

No infarction (TTC) (Plain Lipid Microbubbles)

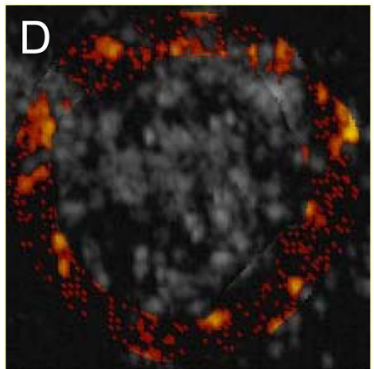

Non-Targeted Microbubbles

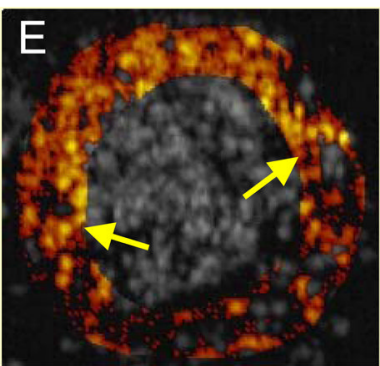

Selectin-Targeted Microbubbles

\section{Figure 4.}

Ischemic memory imaging of myocardium using microbubbles targeted to bind to P-selectin in a rat model of 15 minute coronary occlusion/reperfusion. Short axis ultrasound images of left ventricular myocardium at mid-papillary muscle level are background subtracted, colorcoded frames in which shades of red, progressing to orange, yellow, and white, indicate increasing contrast change. A. During coronary occlusion, there is a contrast defect corresponding to the risk area (region between arrows). B. After release of the coronary occlusion, myocardial contrast echo perfusion imaging with non-targeted lipid bubbles confirms reperfusion to the anterior wall. C. Post-mortem staining with triphenyl tetrazolium chloride (TTC) shows no infarction. D. During reperfusion, imaging after intravenous injection of non-targeted lipid bubbles bearing sialyl Lewis ${ }^{\mathrm{c}}$ as the control ligand, shows no evidence of persistent myocardial contrast enhancement. E. During reperfusion, imaging after intravenous injection of microbubbles targeted to P-selectin via sialyl Lewis ${ }^{\mathrm{X}}$ demonstrates persistent contrast enhancement in the area that was previously ischemic. Reprinted with permission from Ref 27. 

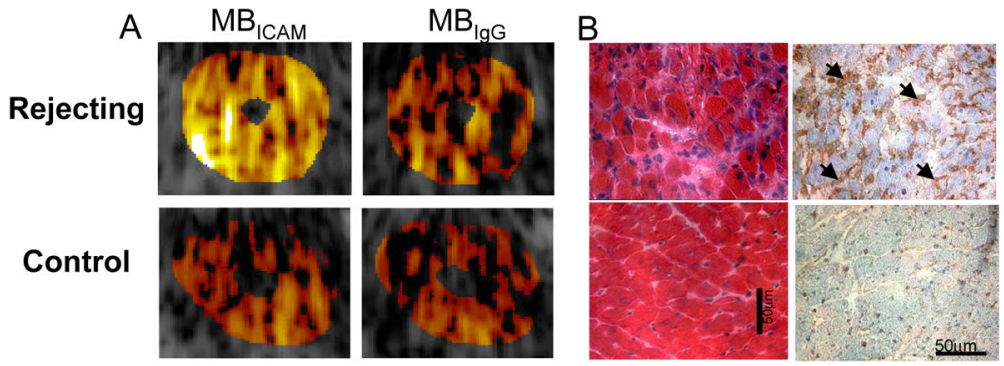

Figure 5.

Detection of acute heart transplant rejection using ultrasound imaging of ICAM1 in rats with abdominal heterotopic heart transplantation. A. Each animal was separately administered microbubbles with anti-ICAM1 antibody ( $\mathrm{MB}_{\mathrm{ICAM}}$, left panels) or isotope control nonspecific IgG ( $\mathrm{MB}_{\mathrm{CTL}}$, right panels) on their surface. In the rejecting allografts (upper panels), $\mathrm{MB}_{\mathrm{ICAM}}$ caused persistent contrast enhancement not seen after injection of $\mathrm{MB}_{\mathrm{CTL}}$. In the non-rejecting isograft hearts (lower panels), there was no significant persistence of myocardial contrast enhancement after injection of either microbubble species. B. Histology of myocardium showing inflammatory infiltrate and ICAM1 (brown) on hematoxylin and eosin and immunohistochemical staining, respectively, in rejecting allograft (upper panels); this was not seen in control isograft myocardium (lower panels). Reprinted with permission from Ref 43. 


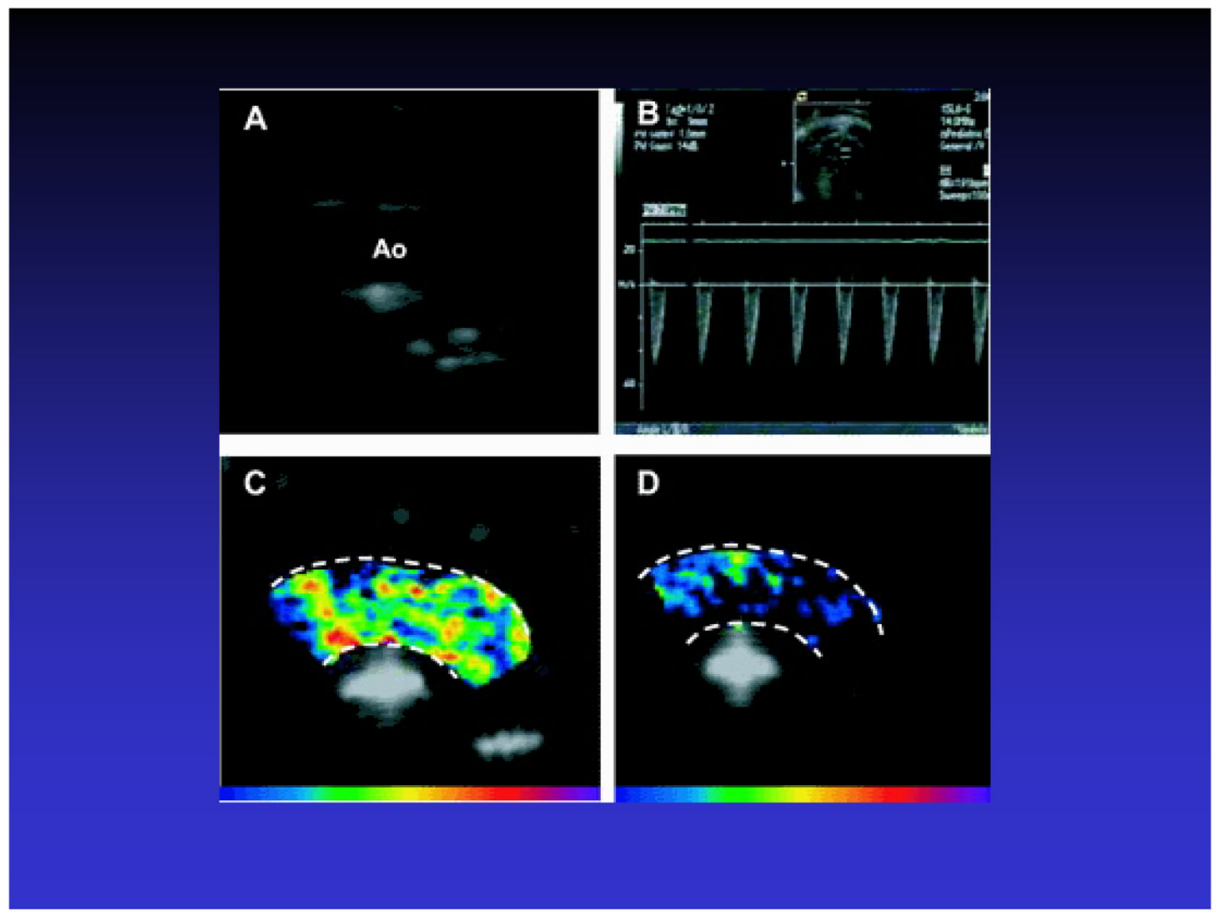

Figure 6.

Ultrasound molecular imaging of early atherosclerosis in Apo E-deficient mice fed a high cholesterol diet. A. Two dimensional imaging of the aortic arch. B. Spectral Doppler imaging of the aortic arch. C. Ultrasound imaging 10 minutes after injection of microbubbles targeted to vascular cell adhesion molecule-1 (VCAM1) via an antibody directed against VCAM-1 demonstrates persistent contrast enhancement of the arch. D. Persistent contrast enhancement of the arch is not seen after injection of control microbubbles conjugated to an isotype control antibody. Reprinted with permission from Ref 44. 


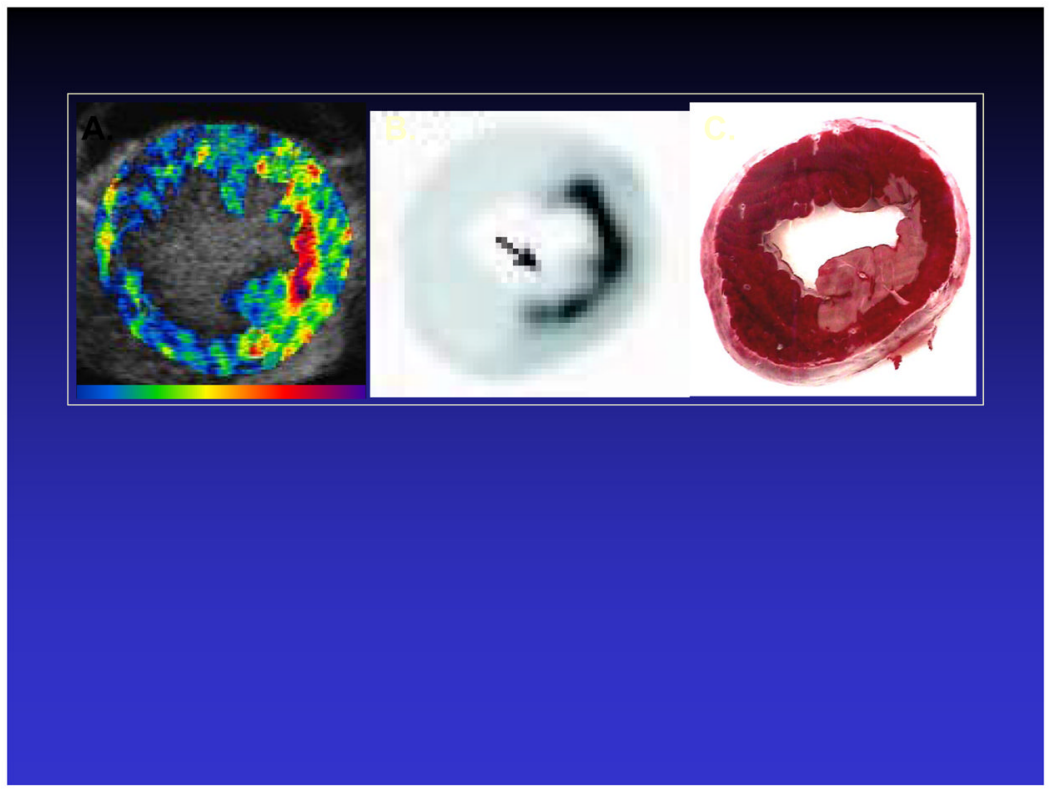

Figure 7.

Inflammatory imaging of post-ischemic/infarcted canine myocardium using activated leukocytes, which have ingested microbubbles, as the molecular probe. A. During reperfusion, there is persistent contrast enhancement in the post-ischemic area. B. The region of enhancement in (A) spatially corresponds to areas of leukocyte activation as shown on autoradiography of isotope-labeled leukocytes (B). C. TTC-stained myocardial section demonstrating area of resulting infarction. Reprinted with permission from Ref 45. 

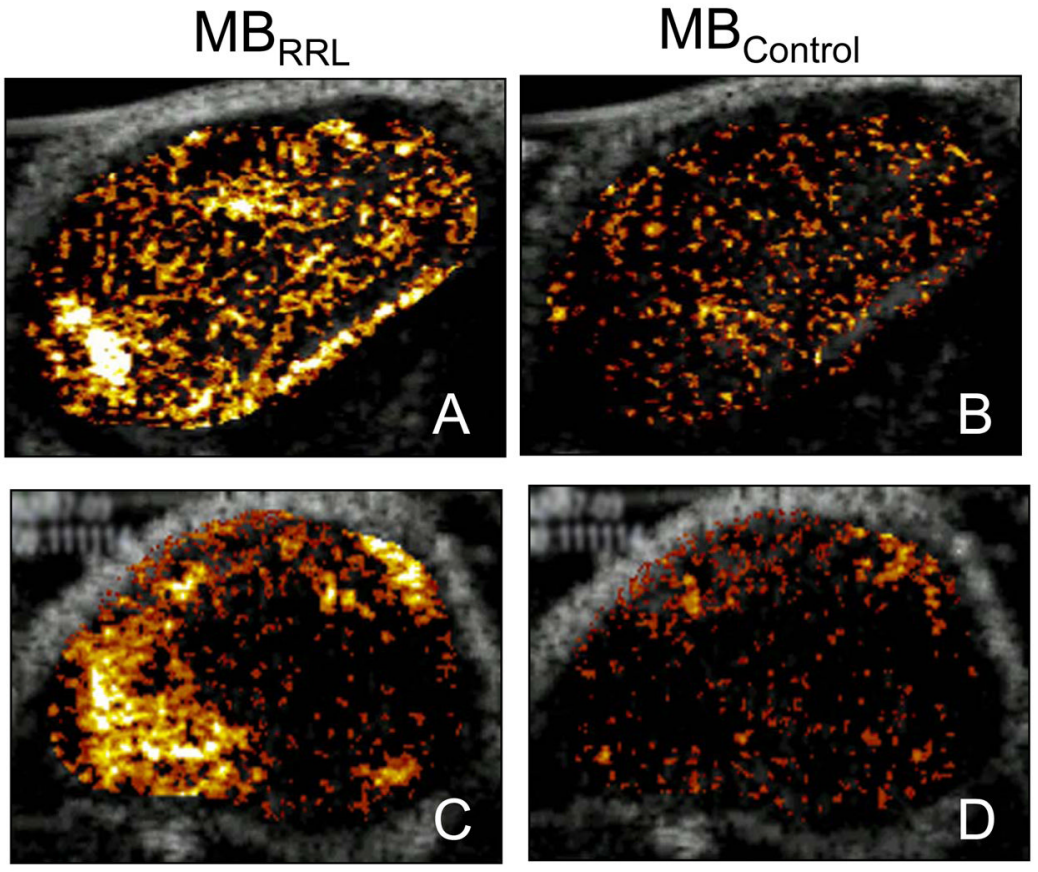

Figure 8.

Ultrasound imaging of angiogenesis using a microbubble conjugated to the tripeptide sequence RRL $\left(\mathrm{MB}_{\mathrm{RRL}}\right)$. Control microbubble $\left(\mathrm{MB}_{\mathrm{CTL}}\right)$ has the sequence $\mathrm{GGG}$ on its surface. Nude mice bearing sarcoma (Clone C) (Panels A and B) or human prostate tumors (PC3) (Panels C and D) were imaged after injection of either $\mathrm{MB}_{\mathrm{RRL}}$ or $\mathrm{MB}_{\mathrm{CTL}}$. There is persistent contrast enhancement of the tumors after injection of $\mathrm{MB}_{\mathrm{RRL}}$ (Panels $\mathrm{A}, \mathrm{C}$ ), which is not seen after injection of $\mathrm{MB}_{\mathrm{CTL}}$ (Panels B, D). Reprinted with permission from Ref 23. 